\title{
Kocuria rosea Bacteremia in Chronic Kidney Disease Patient: A Rare Case Report
}

\author{
Rosy Bala ${ }^{1 *}(\mathbb{D})$, Narinder Kaur ${ }^{1}$ (D), Nitin Gupta² ${ }^{(D)}$ and Udit Narang ${ }^{2}$ (D) \\ ${ }^{1}$ Department of Microbiology, MM Institute of Medical Sciences \& Research, MM Deemed to be University, \\ Mullana, Ambala - 133 207, Haryana, India. \\ ${ }^{2}$ Department of General Medicine, MM Institute of Medical Sciences \& Research, MM Deemed to be \\ University, Mullana, Ambala - 133 207, Haryana, India.
}

\begin{abstract}
Kocuria sp. may cause bacteremia, peritonitis, brain abscesses, meningitis, endocarditis, and acute cholecystitis in immunocompromised individuals. Recent reports identified Kocuria rosea in bacteremia associated with in dwelling intravenous lines, continuous dialysis fluids etc. We report on the case of bacteremia caused by $K$.rosea, a gram-positive microorganism in a 65 -year-old female with a known case of end-stage renal disease on hemodialysis. After Piperacillin and Tazobactam antibiotic treatment, the patient got cured of fever and infection. This report presents a rare case of K.rosea bacteremia successfully treated with common antibiotics. Proper identification systems should be there to know the cause of bacteremia. The bacteremia cases with rare organisms should not be ignored.
\end{abstract}

Keywords: Bacteremia, immune-compromised, Kocuria rosea

*Correspondence: rosy.nitin10@gmail.com; +91 7082708667

(Received: June 22, 2021; accepted: July 29, 2021)

Citation: Bala R, Kaur N, Gupta N, Narang U. Kocuria rosea Bacteremia in Chronic Kidney Disease Patient: A Rare Case Report. J Pure Appl Microbiol. 2021;15(3):1136-1138. doi: 10.22207/JPAM.15.3.40

C The Author(s) 2021. Open Access. This article is distributed under the terms of the Creative Commons Attribution 4.0 International License which permits unrestricted use, sharing, distribution, and reproduction in any medium, provided you give appropriate credit to the original author(s) and the source, provide a link to the Creative Commons license, and indicate if changes were made. 


\section{INTRODUCTION}

Sepsis and bacteremia are severe conditions that are associated with high mortality. Bacteremia can be because of many grampositive as well gram-negative organisms ${ }^{1}$. Bacteremia from Kocuria $s p$. is very rare and only a limited number of case reports of infections by Kocuria sp. are available. Therefore, we have performed a Medline search using the key words of Kocuria rosea," " bacteremia," and "hemodialysis" on https://www.ncbi.nlm.nih. gov/pmc/?term=Kocuria+rosea+bacteremia+ hemodialysis and obtain 18 results as on $23 \mathrm{Jul}$ 2021. Out of theses very few pertain to Kocuria rosea related infections.

Genus Kocuria is aerobic gram-positive bacteria belonging to the family Micrococcaceae. These are found in the environment as saprophytes ${ }^{2}$. About 5 species of genus Kocuria are found to be rare pathogens in cases of catheterrelated bacteremia, peritonitis, brain abscesses, meningitis, endocarditis and acute cholecystitis in immune-compromised individuals ${ }^{3}$.

Here we report a chronic renal disease patient with a bloodstream infection that, after culture, was caused by Kocuria rosea.

\section{Case Report}

A 65 year female with end stage chronic kidney disease; on hemodialysis for past three years was admitted with a complaint of dyspnea and altered sensorium in the Department of Medicine MMIMSR Hospital, Mullana. The patient was a known case of type II diabetes mellitus and hypertension. Clinical, radiological and laboratory diagnostic procedures were performed.

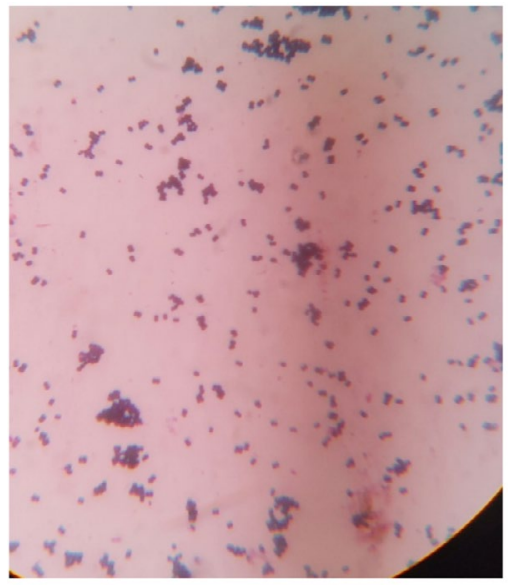

Fig. 1. Gram-stained smear from the growth showing Gram Positive cocci (1000X).

\section{On clinical examination}

Temperature $98.8^{\circ} \mathrm{F}$, blood pressure $150 / 80 \mathrm{mmHg}$, pulse $98 / \mathrm{min}$, respiratory rate $24 /$ min, GCS: E2V2M4, Pallor:++, B/L Pedal Edema: present, JVP: Raised, B/L crepts: present.

\section{Laboratory findings}

Complete blood counts showed anemia ( $\mathrm{Hb}=6.6 \mathrm{gm} \%), \mathrm{TLC}=10,540 /$ cumm and Liver function tests and blood chemistry were within normal limits. ABG showed mild metabolic acidosis, and renal function tests were markedly deranged.

The chest radiograph showed mild pulmonary edema.

On the third day of her admission to the hospital, she developed a fever of $102^{\circ} \mathrm{F}$. RT-PCR for COVID-19: negative. (As the COVID-19 pandemic was going on, the test was done to rule out COVID as the cause of fever.)

Two sets of blood samples were drawn from two different peripheral veins and one time urine sample was collected taking aseptic precautions. The samples were examined for microbial growth using suitable media as per standard protocol. The urine culture report was negative. However, both the blood cultures showed the growth of gram-positive cocci (Fig. 1), which on identification using VITEK 2 system (bioMerieux Inc., France) came to be Kocuria rosea. The antibiotic sensitivity using the VITEK-2 system reported the organism sensitive to all the antibiotics (Penicillins, amoxyclav, piperacillin+tazobactam, amikacin, ciprofloxacin, levofloxacin, erythromycin, clindamycin, linezolid, vancomycin, trimethoprim/sulfamethoxazole etc.).The patient was empirically given the antibiotic injection of Piperacillin+Tazobactam ( $2.25 \mathrm{~g}$ every $8 \mathrm{~h}$ for 14 days) after taking the samples for cultures.

Blood sample was taken for repeat culture after obtaining the first blood culture reports, which became negative as the patient was already receiving the antibiotics. The fever spikes started reducing on the third day of antibiotics and entirely resolved on the fifth day. The patient was discharged in satisfactory condition from the hospital after intravenous piperacillin+tazobactam injections for 14 days.

\section{DISCUSSION}

About 18 different species of gender Kocuria are known. Kocuria $s p$. belong to the 
family Micrococcaceae and are gram-positive cocci $i^{2}$. They are present as saprophytes in the environment or as normal flora on the skin. However, some species can be opportunistic pathogens in humans and rare infections by Kocuria sp. in have been documented. These are generally of low virulence. There might be certain risk factors predisposing the individual to infections by Kocuria species. These risk factors can be: diabetes, metabolic disorders, neoplasms, endstage renal disease, etc. ${ }^{3.7}$. We could found limited literature on $K$. rosea bacteremia. The low number of cases might be because of misidentification as contaminants in the laboratories as coagulasenegative Staphylococci (CoNS). The antibiotic therapy for Kocuria is based on case reports as no clinical guidelines are available for treating such infections. From the available case reports, we found that most of the Kocuria isolates were susceptible to commonly used antibiotics like penicillins, tetracycline, erythromycin, vancomycin, and streptomycin gentamycin, ceftriaxone, chloramphenicol etc. ${ }^{5,8}$.

In conclusion, although Kocuria is an uncommon cause of bacteremia among immunocompromised patients, the occurrence of unusual pathogens from hospitalized patients might not be ignored as contaminants/commensals. As such, ignored strains can be a vector of transfer of drug resistance or resistant strains that can cause of treatment failure with usual antibiotics. Therefore, accurate identification and susceptibility testing with automated identification systems and molecular methods are imperative for diagnosing and treating unusual pathogens.

\section{ACKNOWLEDGMENTS}

None.

\section{CONFLICT OF INTEREST}

The authors declare that there is no conflict of interest.

\section{AUTHORS' CONTRIBUTION}

All authors listed have made a substantial, direct and intellectual contribution to the work, and approved it for publication.

\section{FUNDING}

None.

\section{DATA AVAILABILITY}

The datasets generated during and/or analysed during the current study are available from the corresponding author on reasonable request.

\section{ETHICS STATEMENT}

The study was approved by the institutional Ethics Committee, Maharishi Markandeshwar Institute of Medical Sciences \& Research, Mullana, Ambala, India (Project No: IEC- 159P).

\section{INFORMED CONSENT}

Written informed consent was obtained from the patient for publication of this case report and any accompanying images.

\section{REFERENCES}

1. Christaki E, Giamarellos-Bourboulis EJ. The complex pathogenesis of bacteremia: from antimicrobial clearance mechanisms to the genetic background of the host. Virulence. 2014;5(1):57-65. doi: 10.4161/ viru.26514

2. Stackebrandt E, Koch C, Gvozdiak O, Schumann P. Taxonomic dissection of the genus Micrococcus: Kocuria gen. nov., Nesterenkonia gen. nov., Kytococcus gen. nov., Dermacoccus gen. nov., and Micrococcus Cohn 1872 gen. emend [published correction appears in Int J Syst Bacteriol 1996 Jan;46(1):366]. Int J Syst Bacteriol. 1995;45(4):682-692. doi:10.1099/0020771345-4-682

3. Altuntas F, Yildiz O, Eser B, Gundogan K, Sumerkan B, Cetin M. Catheter-related bacteremia due to Kocuria rosea in a patient undergoing peripheral blood stem cell transplantation. BMC Infect Dis. 2004;4(1):62. doi: 10.1186/1471-2334-4-62

4. Serefhanoglu K, Oklu E. Kocuria rosea bacteremia: two case reports and a literature review. Archives of Medical Science - Civilization Diseases. 2017;2(1):121124. doi: 10.5114/amscd.2017.70601.

5. Purty S, Saranathan R, Prashanth K, et al. The expanding spectrum of human infections caused by Kocuria species: a case report and literature review [published correction appears in Emerg Microbes Infect. 2013 Dec;2(12):e91]. Emerg Microbes Infect. 2013;2(1):1. doi:10.1038/emi.2013.71

6. Paez T, Parra G, Goyes A, et al. Pneumonia by Kocuria rosea: case report and literature review. Pneumologia. 2019;68(1):37-40. doi: 10.2478/pneum-2019-0007

7. Dave VP, Joseph J, Pathengay A, Pappuru RR. Clinical presentations, management outcomes, and diagnostic dilemma in Kocuria endophthalmitis. J Ophthalmic Inflamm Infect. 2018;8(1):21. doi: 10.1186/s12348018-0163-6

8. Szczerba I. Susceptibility to antibiotics of bacteria from genera Micrococcus, Kocuria, Nesterenkonia, Kytococcus and Dermacoccus. Med Dosw Mikrobiol. 2003;55(1):75-80. 